\title{
Diagnostic efficacy of cone beam computed tomography for the TMJ arthropathies
}

\begin{abstract}
The CBCT has been developed recently, to be used specifically in the maxillofacial region, and has quickly positioned itself as the diagnostic imaging modality in most clinical situations, and is one of the elements that make up the evaluation of patients with temporomandibular joint arthropathies. The objective of this article is to discuss the diagnostic efficacy of cbct in the current scenario of the diagnostic imaging of TMJ.

Keywords: cone beam computed tomography, temporomandibular joint disorder, temporomandibular joint imaging, TMJ arthropathies, osteoarthritis, osteoarthrosis, erosion, flattering, osteophyte, subcortical sclerosis, synovial chondromatosis, loose calcified bodies, subcondral cysts
\end{abstract}

Volume II Issue 6 - 2020

\author{
Víctor Pardo Juárez \\ Department of Oral and Maxillofacial Radiology, Scientific \\ University of the South, Peru
}

Correspondence: Víctor Pardo Juárez, Department of Oral and Maxillofacial Radiology, Scientific University of the South, Peru, Email drvictorardo@gmail.com

Received:September 21, 2020 | Published: December 30, 2020
Abbreviations: $\mathrm{CBCT}$, cone beam computed tomography; $\mathrm{CH}$, coronoid hyperplasia; $\mathrm{CT}$, computed tomography; FOV, field of view; MRI, magnetic resonance imaging; OA, osteoarthritis; RA, rheumatoid arthritis; SC, synovial chondromatosis; TMD, temporomandibular disorders; TMJ, temporomandibular joints

\section{Introduction}

Disorders that affect the temporomandibular joint are common, they can affect up to $28 \%$ of the population at some time in their life. They are a heterogeneous group of disorders, classified into two myogenic and arthrogenic groups, although they often overlap. ${ }^{1}$ The joint causes of TDM, and that may affect one or both temporomandibular joints include internal joint disc disorder, acquired degenerative bone defects, inflammatory arthropathies and to a lesser extent neoplastic alteration. . $3,4^{2}$

TDMs consider a subdivision of musculoskeletal pathologies and are the main source of orofacial pain of non-dental origin. ${ }^{5}$ The most common clinical signs and symptoms of TMD are joint pain, muscle pain, limitation of mouth opening, sounds commonly described as a "click", "pop", "crepitation" or "crepitus". Therefore, temporomandibular dysfunction is frequently associated with degenerative bone defects, which affect the bone structures of TMJ. The knowledge of these bone changes is essential for the correct diagnosis of the dysfunctions associated with this disease and for making an adequate treatment plan. ${ }^{6,7}$

There are opinions that say that osteoarthritis is inevitable and almost all patients over 50 years of age will be affected in certain measures. Although TMJ is less problematic for osteoarthritis than the joints of other parts of the body; Studies indicate that TMJ osteoarthritis is found in $40 \%$ of older adults microscopically and $14 \%$ at radiographic levels. ${ }^{8}$ However, although osteoarthritis is definitely an alteration of aging, recent research identified osteoarthritis in the majority of young patients referred to a TMJ clinic for pain and joint dysfunction. ${ }^{9}$ Investigations showed that radiographic osteoarthritic changes of TMJ were common in adolescents and young adults under 30 , especially in women, and the presence frequently important between the ages of 11 to 19 years. ${ }^{10}$
In many cases, the imaging test of TMJ by $\mathrm{CBCT}$ is a decisive factor for the differential and definitive diagnosis of several TMJ alterations. ${ }^{11}$ For the diagnosis by images through a CBCT study of a patient with TMJ arthropathies it is important to take into account fundamental aspects, such as starting with a good clinical examination and an adequate clinical history, in addition to some other important criticisms to evaluate this type of patients that we will see next. ${ }^{12}$

The use of CBCT as a TMJ test was published for the first time, only a couple of years after this modality of maxillofacial imaging will be published in the literature..$^{13}$ The first study evaluated in 2009 and this is used to evaluate the bone components of TMJ, provides high resolution multiplanar images. The $\mathrm{CBCT}$ allows the examination of the anatomy of TMJ without overlapping or distortion to facilitate the analysis of bone morphology, joint space and dynamic function in all three dimensions. ${ }^{14}$

CBCT provides essential information to aid in the diagnosis of a variety of osteogenic and osteolytic TMJ disorders, including osteoarthritis, osteoarthrosis, inflammatory arthritis, trauma, growth and development disorders, and neoplasms (Figures 1-3). ${ }^{15}$

\section{The study of TMJ using ionizing radiation}

Any study using ionizing radiation requires the protection of patients that must be justified, optimized and minimized. When looking for a diagnosis using ionizing radiation, it is essential to maintain the balance between the potential gain of the information and the risk to the patient of exposure to ionizing radiation. The rule is known as "ALARA" for its acronym in English as low as reasonably achievable summarizes this concept and involves the achievement of good diagnostic images with a minimum of radiation exposure.

There are some biological risks of ionizing radiation that should be known: Some radiation effects are deterministic (depending on the amount of radiation used) and some are stochastic (having no limit, leading to changes in cell structures, and genetic modification in the chromosomes, therefore, the genetic modification in the genome of the offspring, etc.). The amount of radiation absorbed in traditional intraoral radiography in the maxillofacial area is relatively small. On the contrary, the use of multislice CT can result in a much greater 
exposure to ionizing radiation; Therefore, the $\mathrm{CBCT}$ provides a substitute for multislice $\mathrm{CT}$, especially in dentistry. $\mathrm{CBCT}$ equipment usually uses a pulsed beam as a kilovolt strobe effect to reduce the effective radiation dose. Thus, for example, during a scan lasting 20 seconds, the patient is exposed to ionizing radiation for only about 3.5 seconds, it has been estimated that the total effective radiation dose is of the order of $0.035-0.10(\mu \mathrm{Sv})$; according to the radiographic technique, the size of FOV and the type of equipment used. This is roughly equivalent to 2 to 8 conventional panoramic radiographs that is much smaller than a maxillary and jaw multi-slice tomography, which is equivalent to 200 to 300 conventional panoramic radiographs. ${ }^{16-27}$

CBCT technology has a very good representation in hard tissue images, it is less expensive, it is widely available, the radiation dose is lower, the amount of radiation varies depending on the brand of the device and the structure to scan and FOV size (Figure $4 \&$ Figure 5). ${ }^{28}$
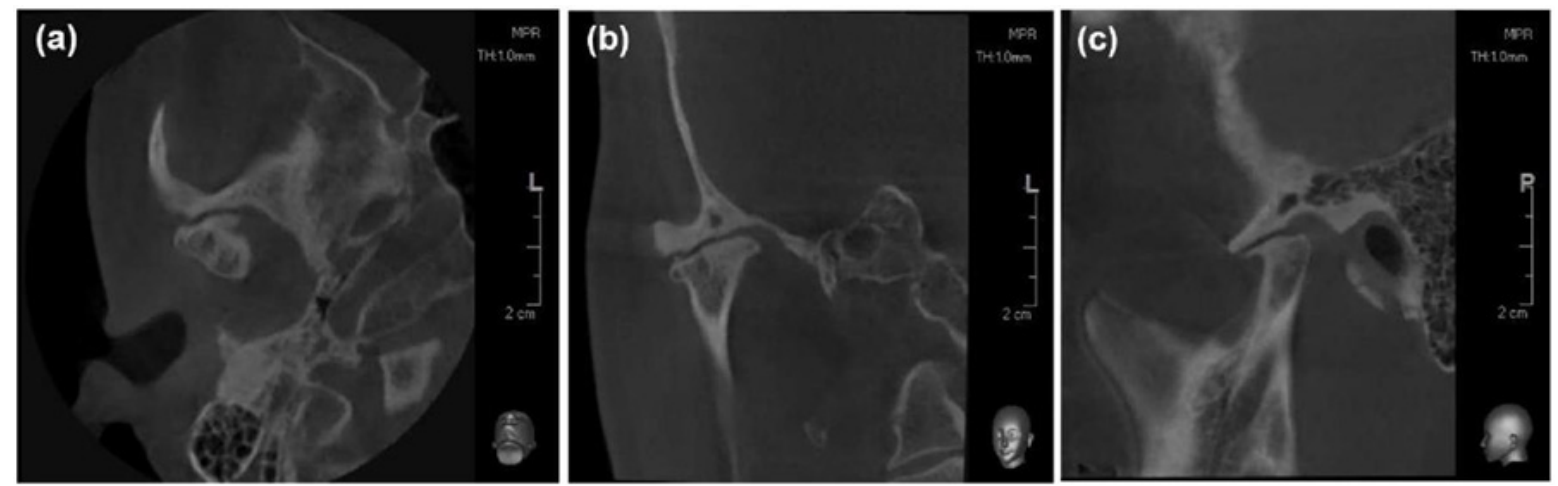

Figure I TMJ in the closed position. Osseous changes characteristic of osteoarthritis. (A) Axial view. (B) Corrected frontal. (C) Corrected sagittal. Note severe flattening of the antero-superior surface of the condylar head, bone sclerosis, erosion, osteophyte formation, subcondral cysts and reduced joint space. (Images was acquired using 8X8 FOV CBCT (Pax-i 3D,Vatech Ewoo Corea).
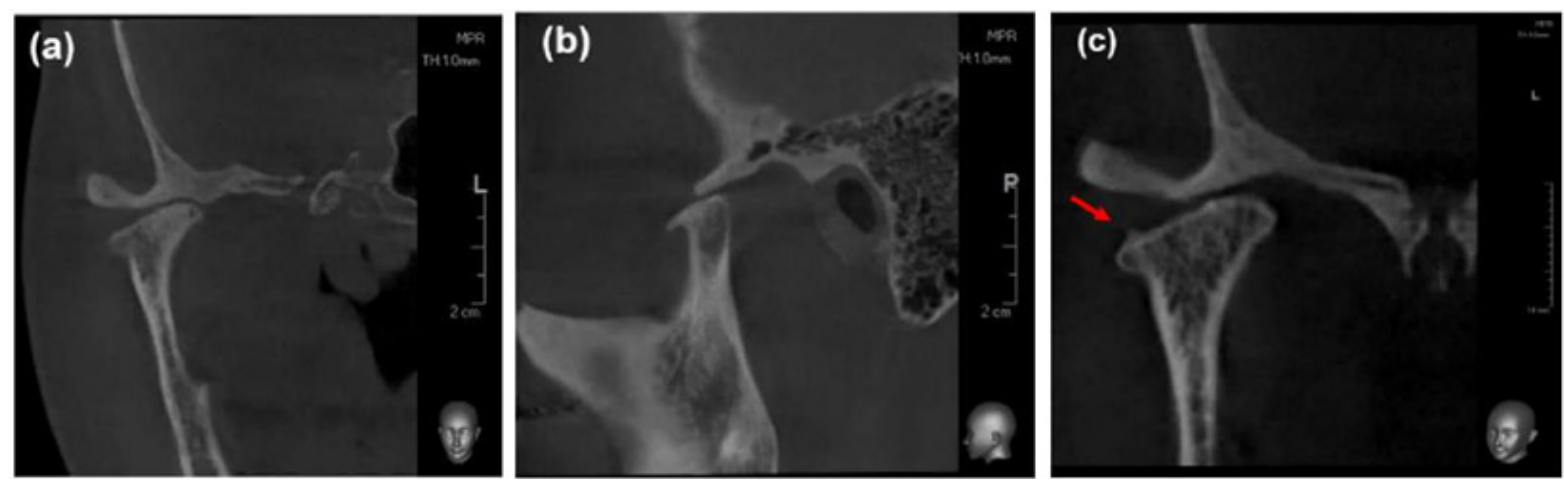

Figure 2 (A) Corrected frontal. (B) Corrected sagittal. Show the position of the condyle-fossa in the open position. (C) Corrected frontal. Note osteophyte formation on superior surface of the condylar head.

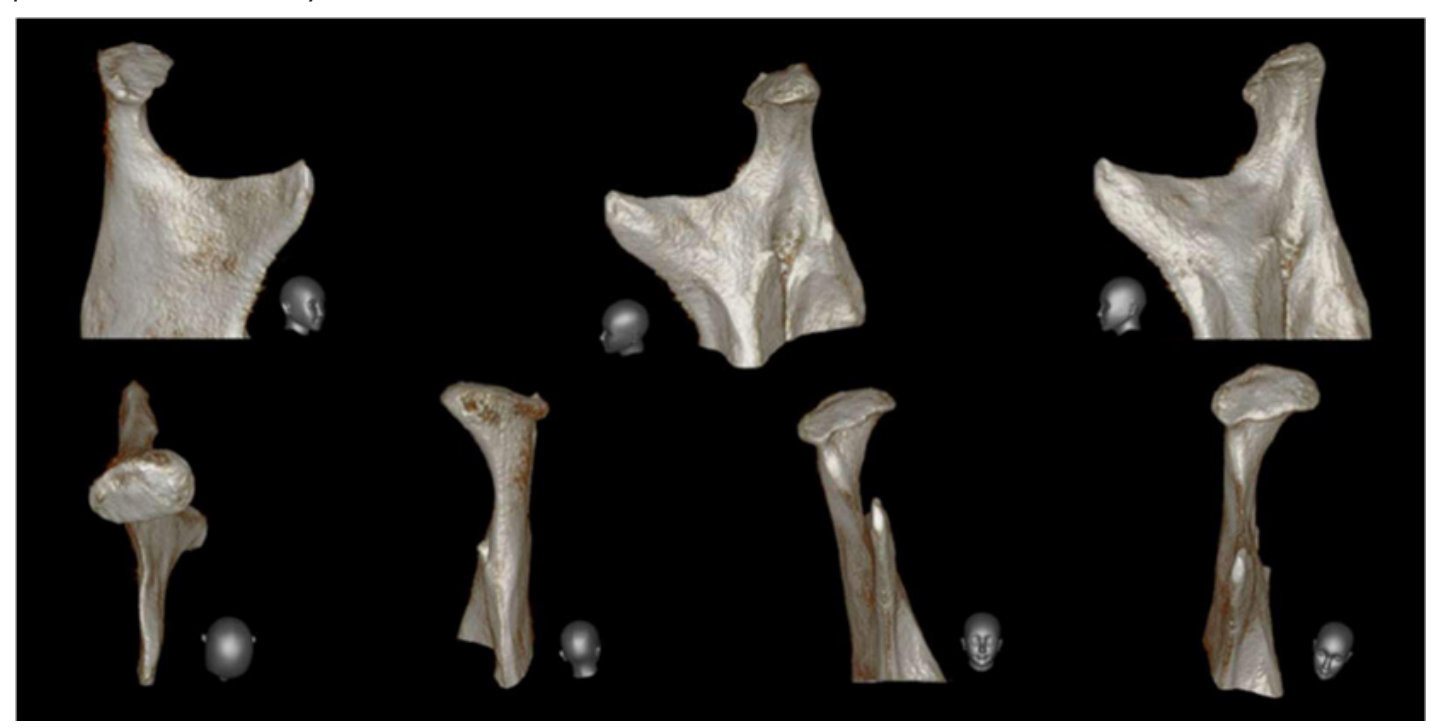

Figure 3 The three-dimensional reconstructions of the right condyle. These representations make it possible to recognize and distinguish in a didactic way the valuable information about the prevailing anatomy, the extent of degenerative bone changes, especially for the examination of this anatomically complex region. 

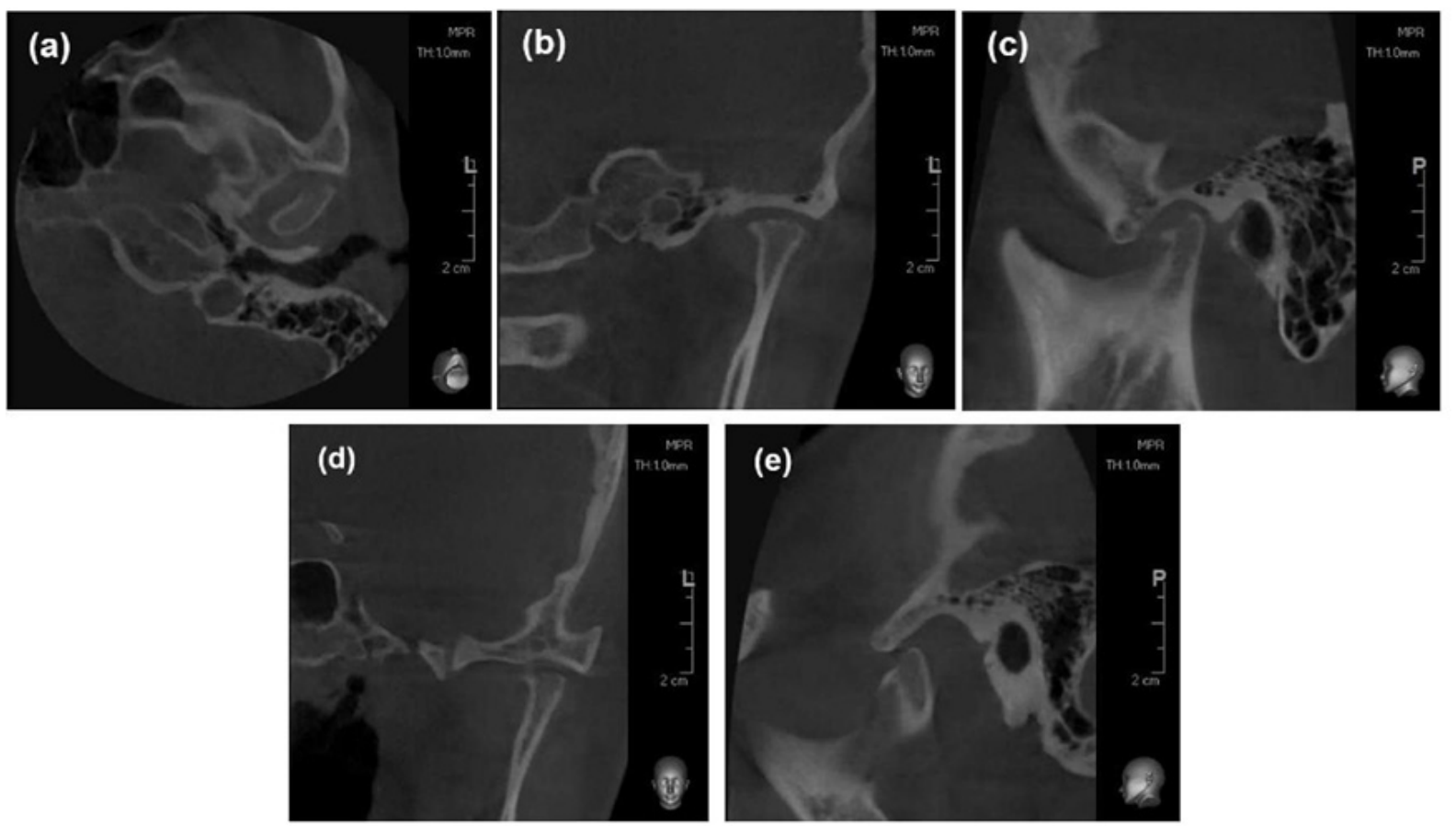

Figure 4 ( $A, B \& C)$ CBCT scans of the TMJ in the closed position, showing osseous changes characteristic of osteoarthritis. Axial view (B) Corrected coronal. (C) Corrected sagittal. Show severe erosion of the antero-superior surface and retruded condylar position in the closed-mouth position. (D) Corrected coronal in open mouth. (E) Corrected sagittal. Limited translational movement of the condyle upon opening (Hypomobility). Images was acquired using 8X8 FOV CBCT (Pax-i 3D,Vatech Ewoo Corea).

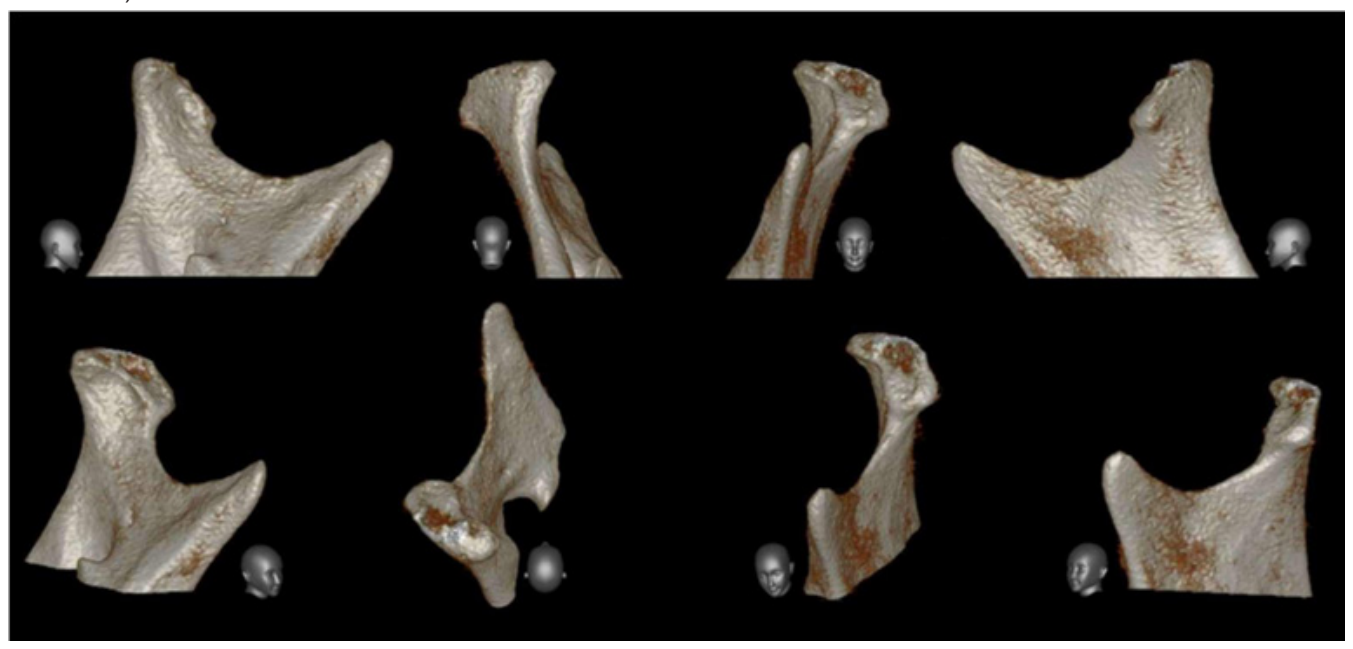

Figure 5 Through three-dimensional reconstructions, the acquired degenerative bone defect that prevails in the head of the condyle can be identified didactically.

\section{Objectives of the TMJ study using CBCT}

The CBCT has contributed to increasing the accuracy in the diagnosis of degenerative bone changes in TMJ. Previous studies have affirmed the diagnostic efficacy of CBCT as it provides accurate and reliable measures for the reconstruction of dental and maxillofacial structures, high spatial resolution, high resolution multiplanar images, therefore, it has demonstrated superior results for the study of TMJ. ${ }^{29}$ An imaging diagnosis is an integral part of the routine clinical evaluation of TMJ conditions and the main objective is to evaluate the integrity of bone structures, when degenerative bone changes are suspected, osteoarthritis, osteoarthrosis, (sclerosis, bone erosions, joint osteophytes, synovial chondromatosis, condylar remodelling, flattening,) or assessing the position of the condyle within the glenoid cavity (relation condyle-fossa), the extent of the condylar excursion, confirming the extent and stage of progression of these disorders, evaluate the effects and evolution of the treatment. Some other conditions of TMJ that we have to keep in mind during the diagnosis are condylar fractures, neoplasms, malformations, remodelling of the roof of the glenoid cavity, pneumatisation of the mastoid cells, potential solution of continuity of the glenoid cavity to the cranial vault, inclination of joint eminence, ankyloses, among others.

Previous research suggests that when referring to degenerative bone changes in TMJ, they should not be studied separately, since one joint influences the other, since, the movements of the jaw are 
coordinated by both joints, being functionally linked, and This functional union could justify the existence of a high prevalence of bilateral degenerative bone changes, in addition, which are significantly more frequent in the condyle than joint eminence. 32 A high prevalence of condylar degenerative changes has also been reported in patients with internal disorders. ${ }^{33}$

Arthritis is an inflammation of the joint surfaces; The two types of arthritis that can affect TMJ are osteoarthritis and osteoarthritis. Studies suggest that pain in the temporomandibular region is twice as common in women than in men; The higher incidence in women can be explained by the hormonal influences of estrogen and prolactin, which can exacerbate the degradation of cartilage and joint bone, in addition to stimulating a series of immunological responses in TMJ. ${ }^{33}$

The most common acquired disorder is osteoarthritis and bone changes associated with this disorder include irregular cortical erosions, osteophytes and subchondral cysts, flattening, free bodies and sclerosis. ${ }^{34}$

The frequency, progression and severity of degenerative bone changes in the condyle and the glenoid cavity are more common in the elderly, due to the development of TMJ osteoarthritis than younger individuals. These degenerative bone changes are considered radiological findings of osteoarthritis and have been demonstrated in TMJ with pain. This has been corroborated in previous studies using $\mathrm{CBCT}$, in patients who had bruxism, and erosion, flattening and osteophytes were found to be the most common radiographic finding of the condyle; The high prevalence of flattening can be explained by the possibility that this degenerative bone change is an adaptive alteration, it is the change of a progressive disease or degenerative change secondary to internal derangement. ${ }^{35}$

Pneumatization of the temporal mastoid cells can be a diagnostic challenge when evaluating cortical erosions in joint eminence if they reach the articular surface, and $\mathrm{CBCT}$ is the most effective method to describe such anatomical variations. The treatment plan has changed by more than $50 \%$ when it is based on clinical exams, panoramic $\mathrm{Rx}$ and CBCT compared to a decision based only on clinical exams and panoramic Rx; In addition to these advantages, the $\mathrm{CBCT}$ provides a three-dimensional image of maxillofacial bone structures with minimal distortion. ${ }^{36}$ In addition to this, the small FOV CBCT is superior to multislice $\mathrm{CT}$ to visualize bone changes in the TMJ, analyzing the transaxial cuts in isolation and combining coronal, axial and sagittal views, Therefore, the CBCT is the method of Ideal image for the diagnosis of TMJ (Figure $6 \& 7$ ). ${ }^{37}$

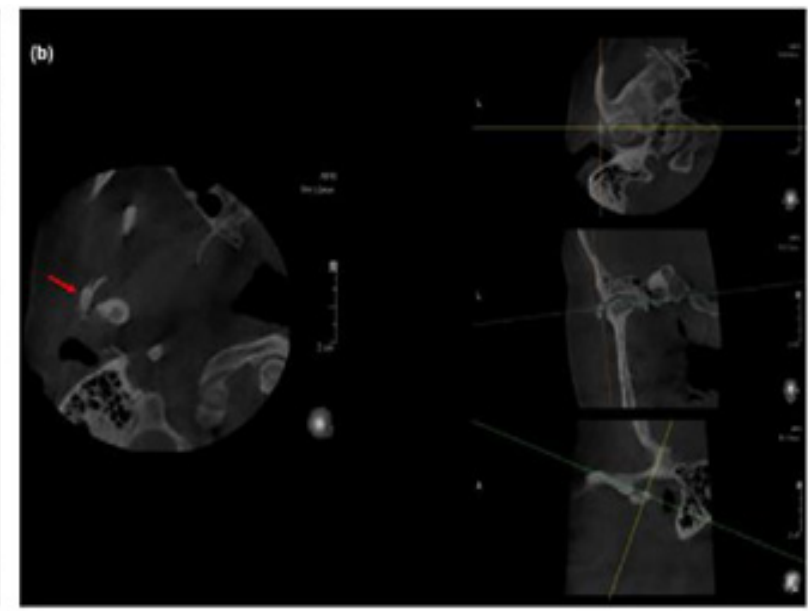

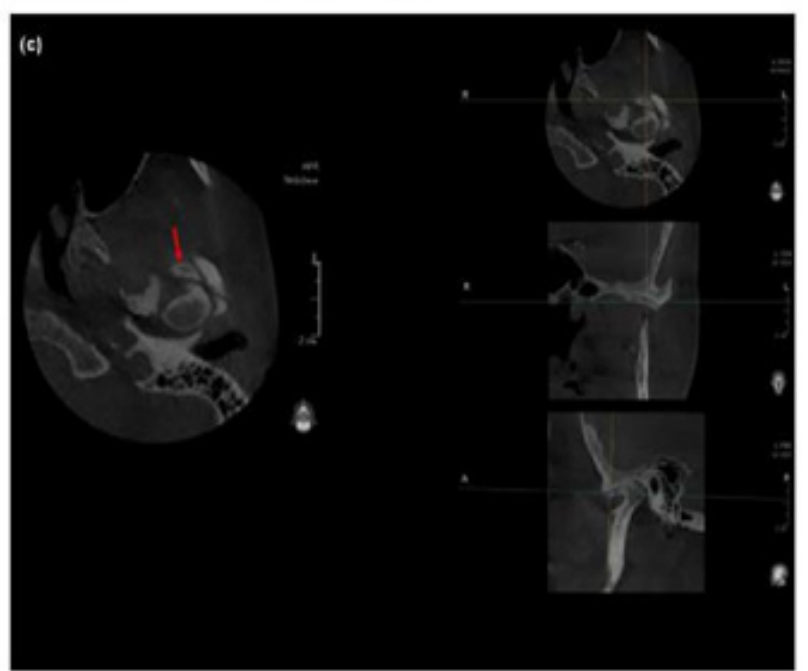

Figure $6(A, B \& C)$ Axial view show the location of the three free bodies through the axes of rotation in multiplanar views. 


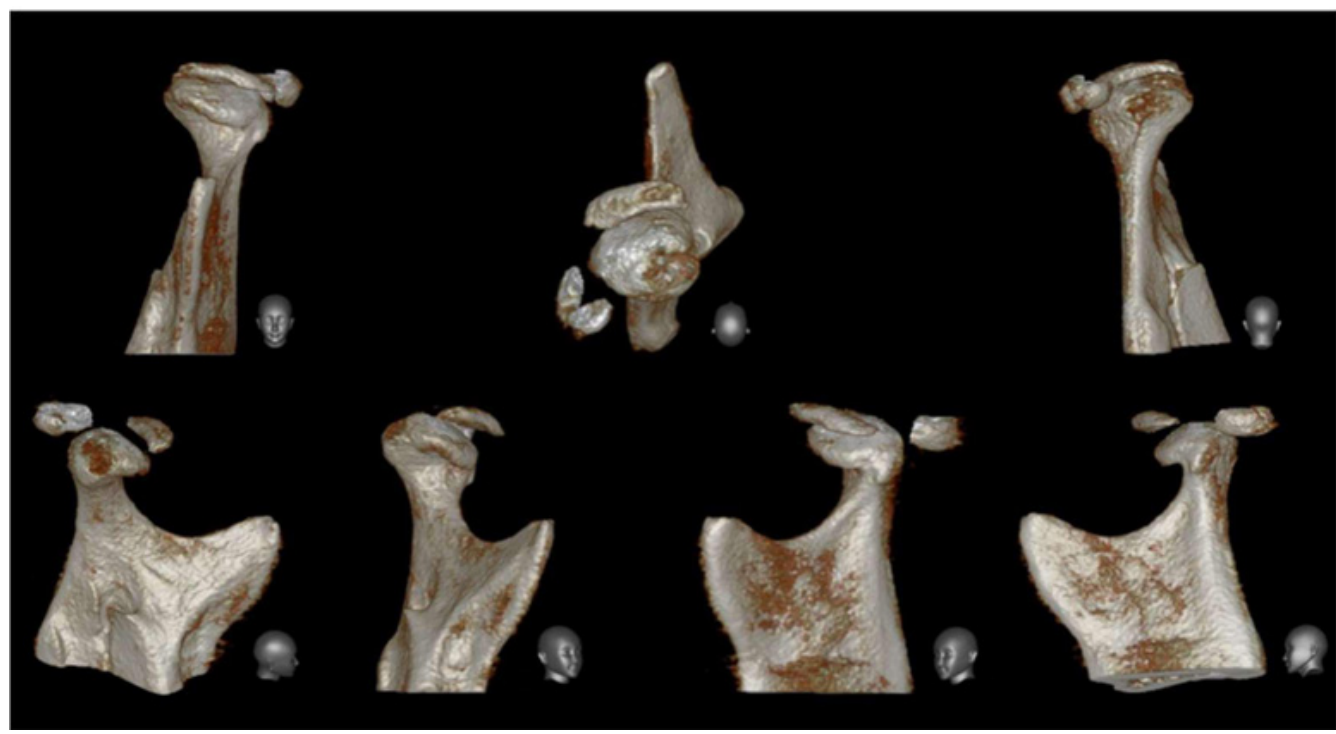

Figure 7 Communication with patients is often definitive to understand the treatment plan and sight through three-dimensional reconstructions is a good method for that purpose.

\section{Description of degenerative bone changes of TMJ}

Osteoarthritis. Also called degenerative arthropathy; it is when the bone alterations are active; This is a progressive, heterogeneous disease that causes joint degeneration is often painful and limits daily activities, including talking and chewing; and the symptoms are accentuated with mandibular movements. Osteoarthritis can appear at any time when the joint suffers an overload, but it is more common in case of dislocation or disc perforation; Once disc dislocation occurs and retrodiscal tissues rupture, the condyle comes into contact directly with the glenoid fossa, accelerating the degenerative process. Over time, the dense fibrous joint surfaces are destroyed and bone changes occur; Any movement causes pain, so the jaw function is quite restricted. Etiology. The most frequent etiological factor that contributes to produce it is the overload of the articular structures. Apparently, this disorder is not a true inflammatory response; Rather, it is a non-inflammatory disorder, where joint surfaces and underlying bone deteriorate. The exact cause is unknown, but in general it is thought to be due to mechanical joint overload; The overload of the articular surfaces may be due to a high level of parafunctional activity coupled with a malocclusion due to discrepancy of the jaws, especially when the articular structures are not properly seated to receive the force. Therefore, osteoarthritis represents a destructive process that It deteriorates the bone joint surfaces of the mandibular condyle and the glenoid fossa. If these overloads are prolonged, the articular surface softens producing an alteration called chondromalacia and the subarticular bone begins to be reabsorbed. With the passage of time, progressive degeneration causes the loss of the subchondral cortical stratum, bone erosion and the consequent radiological evolutionary signs of osteoarthritis. Tomographic signs. This is usually confirmed by CBCT, which will show signs of structural alterations in the mandibular condyle and/or the glenoid cavity called acquired degenerative bone defects. ${ }^{35}$ Erosion. It is the initial phase of degenerative bone changes, which indicates that TMJ is unstable and changes occur in bone surfaces. It is evidenced as a loss of continuity of the articular cortex. Surface flattening: It is a bone contour that creates a flat surface causing a loss of the rounded contour of the surface that deviates from the natural convex shape of the condyle. This bone change represents an adaptive alteration and is the first change of a progressive disease or a degenerative change secondary to internal disorder; This is also considered a degenerative alteration resulting from TMJ overload and may be related to participation in the masseter and temporal muscle overload, and could result in changes in occlusion. Osteophyte They are marginal bone proliferations in the condyle bone; They occur at an advanced stage of degenerative change when the organism adapts to repair the condyle. The osteophyte appears to stabilize and enlarge the surface in an attempt to improve the overload resulting from occlusal forces. It is evidenced as a marginal hypertrophy with sclerotic edges and exophytic angular formation of bone tissue from the surface; It is rare, but it has been reported that this neoformation could fracture and create a free bone body within the joint capsule. Sclerosis. It is the increase in the density of the cortical bone that extends to the bone marrow; Manifest as any increase in thickness of the cortical plate in load areas in relation to adjacent non-load bearing areas.

There is no clear trabecular orientation without delimitation between the cortical plate and the trabecular bone, it could extend to a large part of the condylar head. Synovial chondromatosis. Also called free body, this is due to chondrometaplasia of synovial joint tissues; Cartilaginous nodules form within the synovium and often separate from the synovial membrane, becoming free bodies within the joint space. It usually affects large joints and is relatively uncommon in TMJ. It is believed that synovial chondromatosis is secondary to factors such as trauma, microtrauma or degenerative arthritis. Pseudocyst or subchondral cyst. Also called Ely's cyst, it is a wellcircumscribed osteolytic area of the adjacent subcortical bone without cortical destruction. It is observed as a cavity below the articular surface that deviates from the normal medullary pattern. ${ }^{10,38,39}$

\section{Osteoarthrosis}

Many times, as the overload decreases, the arthritic process becomes an adaptation process, although bone morphology is still affected and this phase of adaptation is called osteoarthrosis. This adaptation process is when a bone remodelling occurs, the dysfunction can be stabilized even though the bone morphology continues to be altered and it is confirmed when the structural alterations of the subarticular bone are observed in the studies, but the patient does not report pain symptoms; although, crepitation is frequent. 


\section{Etiology}

It is caused by joint overload. When the load on the joint is mild, bone remodelling takes place without symptoms. This process is the natural way of adapting to the functional demands of the system. If the functional demands exceed the adaptive capacity, osteoarthritis begins; When the adaptive process has satisfied the functional requirements, it becomes an osteoarthritis. In the background a period of time in which there were symptoms related to osteoarthritis can be identified; The patient may report that the bite has changed over time. Tomographic signs. This disorder is confirmed when structural changes of the subarticular bone are noticed in imaging studies. ${ }^{10,40,41}$ Condylar position (Concentric, Joint compression, Joint distraction,
Joint retrusion). Concentric position. also called centralized, is when the joint spaces are preserved. Joint compression. It is when there is a decrease in the superior and/or anterior joint space. Joint distraction. It is the increase of the upper joint space. Joint Retrusion. It is when there is a decrease in the posterior joint space. Verification of the amplitude of the condylar excursion. (Hypomobility, Normal mobility, Hypermobility). Hypermobility. Mandibular condyle located behind the articular eminence during the upon opening. Normal mobility. Condylar displacement conserved during the upon opening.

\section{Hypermobility}

When the mandibular condyle manages to be located ahead of the articular eminence during the upon opening (Figures 8-10). ${ }^{42-60}$
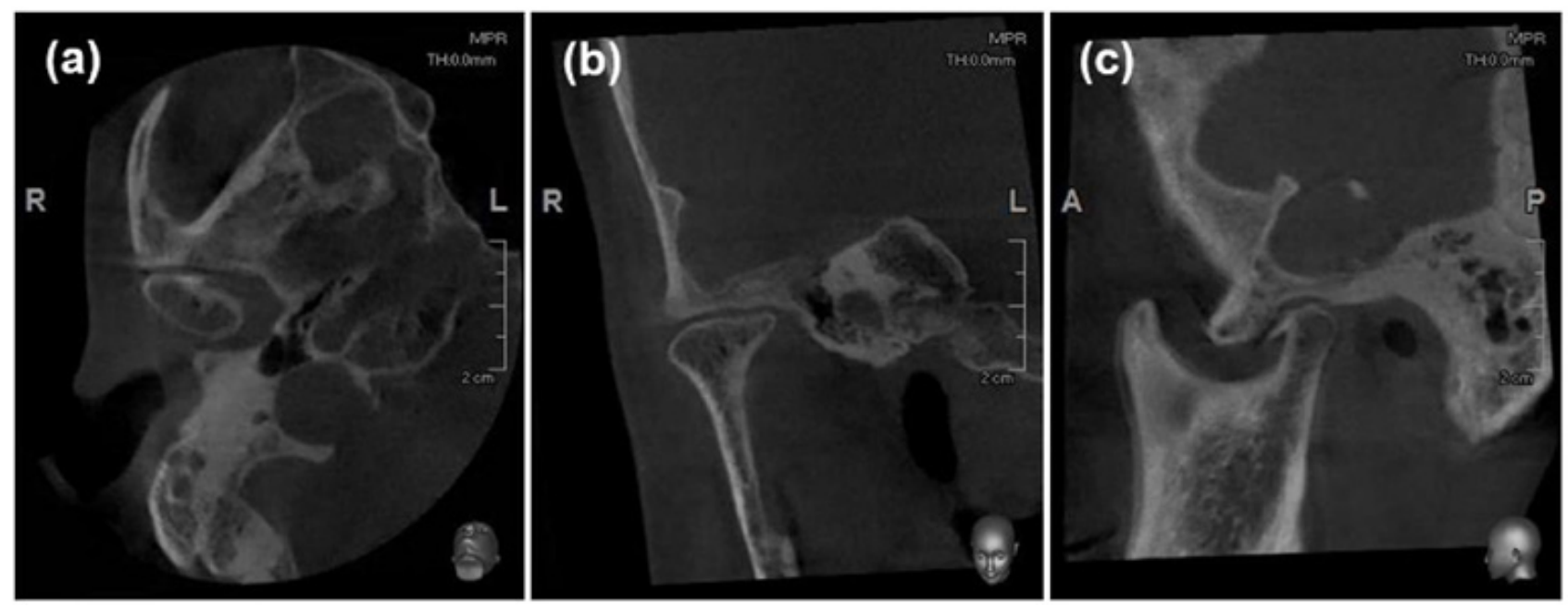

Figure $8 \mathrm{TMJ}$ in the closed position. Osseous changes characteristic of osteoarthritis. (A) Axial view. (B) Corrected frontal. (C) Corrected sagittal. Note that this patient has severe flattening of the antero-superior surface of the condylar head, bone sclerosis of the anterior surface, osteophyte formation, subcondral cysts and reduced joint space. Images was acquired using 8X8 FOV CBCT (Pax-i 3D,Vatech Ewoo Corea).
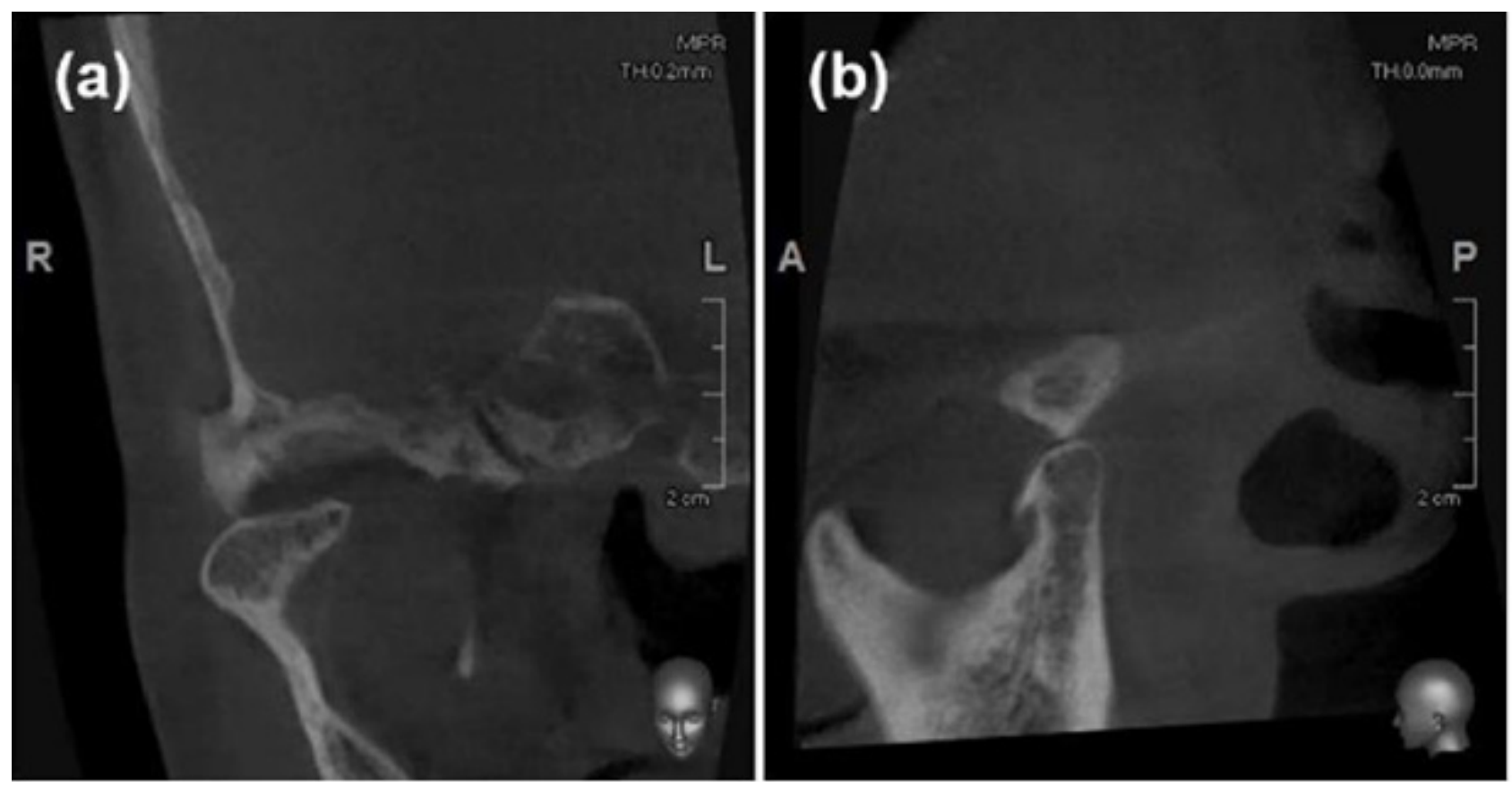

Figure 9 (A) Coronal view. Corrected coronal in open mouth. (B) Corrected Sagittal. The limit of movement of the condyle seems not to be limited upon opening. 


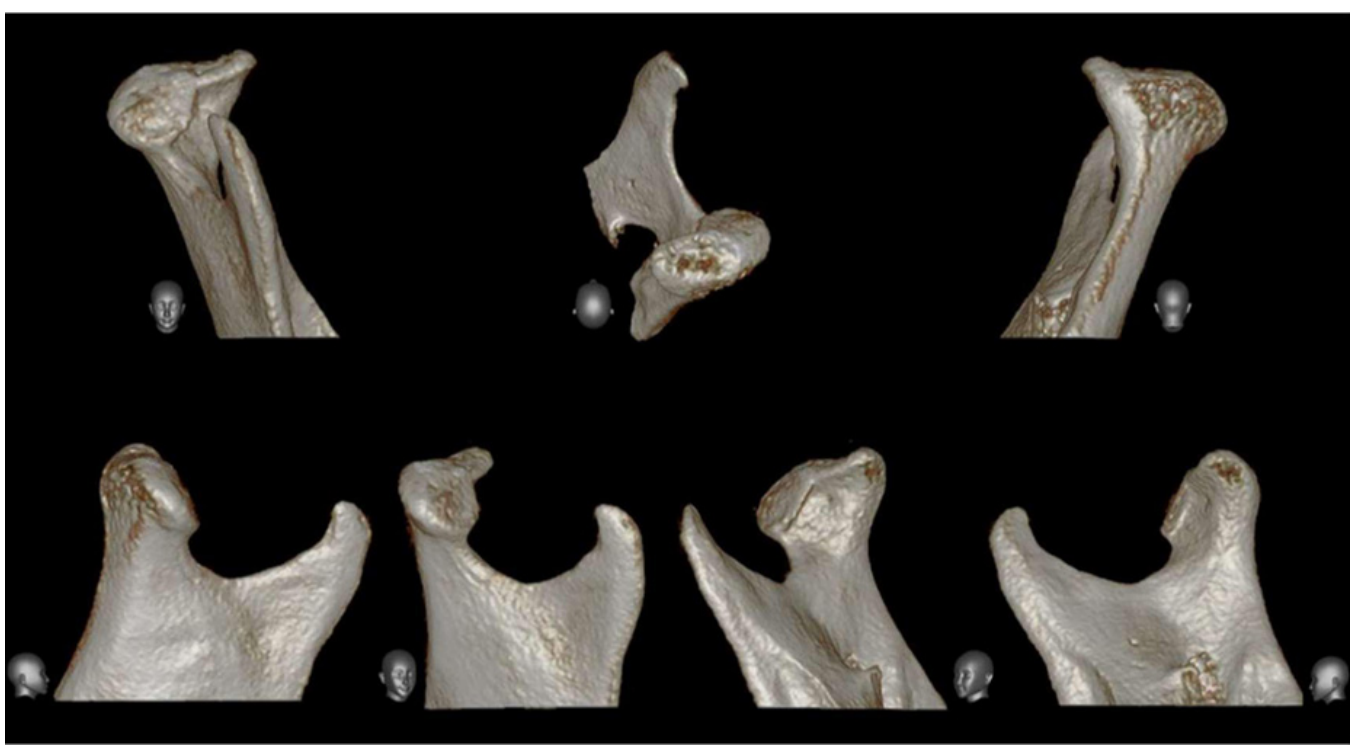

Figure 10 CBCT image showing the right TMJ with three-dimensional reconstruction (3D) and bone defects in the condylar head.

\section{Diagnostic accuracy}

The device emits a conical X-ray beam, instead of a fan-shaped beam used in multislice CT. The equipment revolves around the patient in 10 to 40 seconds depending on the machine, and in a single scan captures images of a cylindrical volume called the field of view. When a large field of view of $15 \times 15$ is used, the majority of the maxillofacial complex enters this field, but there are also FOVs of 21 X19 where most of the entire skull fits most of the time; During this scan the computer collects the information and through very complex mathematical algorithms, it transforms them into small cubes called voxels (which are generally of a $0.3 \mathrm{~mm}$ size) process called primary reconstruction; The size of the voxels in the most recent devices is even smaller $(0.15 \mathrm{~mm})$, which improves spatial resolution.

The equipment with a small FOV provides sufficient anatomical coverage to perfectly cover the TMJ area for example an $8 \mathrm{X} 8$, these high-resolution scans provide an excellent visualization of the TMJ bone changes. Large FOV CBCT Equipment can also be used for the diagnosis of TMJ; Depending on the FOV selected, the image volume can cover both TMJ. However, it is important to note that with a large FOV and higher voxel sizes, the image resolution is reduced and this could compromise the ability to detect early bone changes. ${ }^{45,61}$ Recent studies have shown that CBCT, which is scanned with a smaller size of FOV and voxel, has a higher diagnostic accuracy for the detection of TMJ erosions. Depending on the diagnostic task, the entire maxillofacial skeleton may be necessary, where an evaluation of craniofacial asymmetry or a combination of small FOV and large FOV scans may be necessary for these patients. ${ }^{45,46}$ Studies have shown comparing different sizes of FOV and with different voxel sizes, the highest diagnostic efficacy to identify condylar erosions was found with a smaller FOV, approximately one in three small defects in both diameter and depth, 1 to $2 \mathrm{~mm}$ approximately, they may not be detected in the diagnosis, when large size and large voxel CBCT scans are used. However, using a higher resolution with a Small FOV size and small voxel defects, regardless of size, will be detected with a sensitivity of $80 \%$ depending on the equipment. 46 The evaluation of cortical bone in young individuals is a greater challenge than in adult individuals because cortical bone does not necessarily have to be continuous and compact due to its age. In a CBCT study, it was concluded that cortical bone begins to form around the periphery of the condyle during adolescence: between 12 and 14 years of age. A continuous, homogeneous and compact cortical bone layer on the articular surface is established in young people aged 21 to 22 years, which indicates a complete development of the mandibular condyle. ${ }^{47,48}$ Images with artifacts of movement and with a lot of noise combined with the lack of a continuous and compact cortical layer can make the evaluation of the articular surfaces of young patients uncertain. 48, 49, 62 Bone changes in TMJ can indicate that a pathological alteration is present as osteoarthritis and/or a remodeling process. The boundary between these two anomalies can be difficult to diagnose, especially in growing and developing individuals, in which TMJ is undergoing morphological changes, in these cases an incipient osteoarthritis in young patients can go unnoticed or even over-diagnosed. It has been determined that the reliability of $3 \mathrm{D}$ studies is acceptable through overlay methods for the evaluation and comparison of condylar morphology; The authors affirm that subtle bone differences in condylar morphology can be quantified by $3 \mathrm{D}$ studies. ${ }^{50,51,63}$ Protocols for using and interpreting cbct in TMJ arthropathies. An important aspect is to familiarize yourself with the software that we will use in the interpretation of the TMJ volume. For secondary reconstruction, the axial views on which the condylar processes with their wider mediolateral extension are observed should be used as a reference; 52 The investigation of condylar degenerative bone changes in the axial, coronal and sagittal planes should be performed; The condyle-fossa relationship must be evaluated in the corrected sagittal and corrected coronal view at maximum intercuspidation; that is, the volume of the image can be reconstructed in parallel and perpendicular planes to the long axis of the condyle, instead of the true coronal and sagittal anatomical planes. These reconstructed views also allow a better evaluation of the position of the condyle within the glenoid fossa; The amplitude of the condylar excursion can be interpreted using corrected sagittal view at the maximum oral opening. The panoramic reconstructions generated are not comparable with digital panoramic radiographs, special care must be taken when interpreting them. ${ }^{50,51,64}$

It is important to recognize that the term CBCT does not refer to a single imaging protocol and that the accuracy of diagnostic detection of degenerative bone changes in TMJ has been shown to be influenced 
by the study protocol of the CBCT unit used for the examination, of the size of the defects; extremely small defects, that is, $1 \mathrm{~mm}$ or less, can be difficult to detect; of motion artifacts; FOV size used, voxel size, including patient age. Three-dimensional reconstructions should be used with caution to assess degenerative bone changes. $28 \mathrm{TMJ}$ studies when using a FOV size that is limited exclusively to the TMJ region for example in an $8 \mathrm{X} 8 \mathrm{FOV}$ should therefore be performed bilaterally in 4 scans in total; two bilateral scans in maximum intercuspidation and 2 bilateral scans in maximum aperture. ${ }^{52,53,65}$

\section{Conclusion}

In a short time, $\mathrm{CBCT}$ has emerged as an ideal alternative because of the lower cost and low dose of effective radiation, compared to multislice $\mathrm{CT}$ for the examination of TMJ, although it may be more sensitive to motion artifacts. $30 \mathrm{CBCT}$ is the most effective method to describe such anatomical variations of TMJ. The treatment plan has changed by more than $50 \%$ when it is based on clinical exams, panoramic $\mathrm{Rx}$ and $\mathrm{CBCT}$ compared to a decision based only on clinical exams and panoramic Rx; Therefore, the efficacy of CBCT in the management of patients with bone abnormalities of TMJ is clearly demonstrated; In summary, the studies confirm that the reconstruction technique for imaging using axial, sagittal, coronal CBCT and 3D reconstructions of the ATM had a high diagnostic quality with low dose of effective radiation, therefore, it has become a medium widely available for oral and maxillofacial diagnosis.

\section{Funding}

None.

\section{Acknowledgments}

None.

\section{Conflicts of interest}

The authors declare that there is no conflict of interest to declare.

\section{References}

1. Larheim TA, Abrahamsson AK, Kristensen M, et al. Temporomandibular joint diagnostics using CBCT. Dentomaxillofac Radiol. 2015;44(1):20140235.

2. ML dos Anjos Pontual, JSL Freire, JMN Barbosa, et al. Evaluation of bone changes in the temporomandibular joint using cone beam CT. Dentomaxillofac Radiol. 2012;41(1):24-29.

3. Dilhan Elgüy, Mehmet Ilgüy, Erdogan Fisekçioglu, et al. Articular Eminence Inclination, Height, and Condyle Morphology on Cone Beam Computed Tomography. Scientific World Journal. 2014;761714.

4. Roque-Torres G, Meneses-López A, F Norberto Bóscolo, et al. Cone beam computed tomography use in orthodontics, functional facial orthopedics. Rev Estomatol Herediana. 2015;25(1):60-77.

5. Marinescu-Gava M. What the general dental practitioner should know about cone beamcomputed tomograph technology. OHDMBSC. 2009;8(4):14-21.

6. Zain-Alabdeen EH, Alsadhan RI. A comparative study of accuracy of detection of surface osseous changes in the temporomandibular joint using multidetector CT and cone beam CT. Dentomaxillofac Radiol. 2012;41(3):185-191.

7. Liang X, Jacobs R, Hassan B, et al. Acomparative evaluation of Cone Beam Computed Tomography (CBCT) and Multi-Slice CT (MSCT). Part I. On subjective image quality. Eur J Radiol. 2010;75(2);265-269.
8. Jeffrey $\mathrm{P}$ Okeson. Treatment of occlusion and temporomandibular affections. 5th ed. Madrid: Mosby; 2003.

9. Alexiou KE, Stamatakis HC, Tsiklakis K. Evaluation of the severity of temporomandibular joint osteoarthritic changes related to age using cone beam computed tomography. Dentomaxillofac Radiol. 2009;38(3):141147.

10. Eric Whaites. Dental Radiology. 2nd ed. Buenos Aires: Medica Panamericana; 2010.

11. Bhuvana Krishnamoorthy, Mamatha NS, Vinod Kumar AR. TMJ imaging by CBCT: Current scenario. Ann of Maxillofac Surg. 2013;3(1):80-83.

12. Perez Marques A, Perrella A, Saito Arita E, et al. Assessment of simulated mandibular condyle bone lesions by cone beam computed tomography. Braz Oral Res. 2010;24(4):467-474.

13. Perrella A, Borsatti MA, Peixoto Tortamano I, et al. Validation of computed tomography protocols for simulated mandibular lesions. A comparison study. Braz Oral Res. 2007;21(2):165-169.

14. Boeddinghaus $\mathrm{R}$, Whyte $\mathrm{A}$. Computed tomography of the temporomandibular joint. $J$ Med Imaging and Radiat Oncol. 2013;57(4):448-454.

15. Barghan S, Tetradis S, Mallya SM. Application of cone beam computed tomography for assessment of the temporomandibular joints. Aust Dent $J$. 2012;57(1):109-118.

16. Gorucu Coskuner, Semra Ciger. Three-dimensional assessment of the temporomandibular joint and mandibular dimensions after early correction of the maxillary arch form in patients with Class II division 1 or division 2 malocclusion. Korean J Orthod. 2015;45(3):121-129.

17. Al-Saleh MAQ, Jaremko JL, Alsufyani N, et al. Assessing the reliability of MRI-CBCT image registration to visualize temporomandibular joints. Dentomaxillofac Radiol. 2015;44(6):20140244.

18. Kyung-Soo Nah. Condylar bony changes in patients with temporomandibular disorders: a CBCT study. Imaging Sci Dent. 2012;42:249-53.

19. Suomalainen A, Pakbaznejad Esmaeili E, et al. Dentomaxillofacial imaging with panoramic views and cone beam CT. Insights Imaging. 2015;6(1):1-16.

20. Ozkan A, Altug HA, Sencimen, M, et al. Evaluation of Articular Eminence Morphology and Inclination in TMJ Internal Derangement Patients with MRI. Int. J Morphol. 2012;30(2): 740-774.

21. Katsavrias EG. Changes in Articular Eminence Inclination During the Craniofacial Growth Period. Angle Orthod. 2002;72(3):258-264.

22. Kranjčić J, Vojvodić D, Žabarović D, et al. Differences in particulareminence inclination between medieval and contemporary human populations. Arch Oral Biol. 2012;57(8):1147-1152.

23. Loubele M, Guerrero ME, Jacobs R, et al. A comparison of jaw dimensional and quality assessments of bone characteristics with ConeBeam CT, Spiral Tomography, and Multi-Slice Spiral CT. Int J Oral Maxillofac Implants. 2007;22(3):446-454.

24. Pauwels R, Beinsberger J, Collaert B, Theodorakou C, Rogers J, Walker A, et al, Effective dose range for dental cone beam computed tomography scanners. Eur J Radiol. 2012;81(2):267-271.

25. Suomalainen A, Kiljunen T, Käser Y, et al. Dosimetry and image quality of four dental cone beam computed tomography scanners compared with multislice computed tomography scanners. Dentomaxillofac Radiol. 2009;38(6):367-378.

26. De Vos W, Casselman J, Swennen G. Cone-beam computerized tomography (CBCT) imaging of the oral and maxillofacial region: a systematic review of the literature. Int $J$ Oral and Maxillofac Surg. 2009;38:609-625. 
27. Horner K, Islam M, Flygare L, et al. Basic principles for use of denta cone beam computed tomography: consensus guidelines of the European Academy of Dental and Maxillofacial. Dentomaxillofac Radiol. 2009;38(4):187-195.

28. Underhill TE, Chilvarquer I, Kimura K, et al. Radiobiologic risk estimation from dental radiology. Part I. Absorbed doses to critical organs. Oral Surg Oral Med Oral Pathol. 1988;66(1):111-120.

29. Ngan DC, Kharbanda OP, Geenty JP, et al. Comparison of radiation levels from computed tomography and conventional dental radiographs. Aust Orthod J. 2003;19(2):67-75.

30. Silva MA, Wolf U, Heinicke F, Bumann A, Visser H, Hirsch E. Cone-beam computed tomography for routine orthodontic treatment planning: a radiation dose evaluation. Am J Orthod Dentofacial Orthop. 2008;18:133:640.

31. Choudhary AB, Motwani MB, Degwekar SS, et al. Utility of digita volume tomography in maxillofacial trauma. J Oral Maxillofac Surg. 2011;69(6):e135-e140.

32. De Vos W, Casselman J, Swennen G. Cone-beam computerized tomography $(\mathrm{CBCT})$ imaging of the oral and maxillofacial region: a systematic review of the literature. Int J Oral and Maxillofac Surg. 2009;38(6):609-625.

33. Tsiklaklis K, Syriopouluos K, Stamatakis HC. Radiographic examination of TMJ using cone beam computed tomography. Dentomaxillofac Radiol. 2004;33(3):196-201.

34. Ludlow JB, Davies-Ludlow LE, Brooks SL. Dosimetry of two extraoral direct digital imaging devices: NewTom cone beam CT and Orthophos Plus DS panoramic unit. Dentomaxillof Radiol. 2003;32(4):229-234..

35. Pauwels, Jilke Beinsberger, Bruno Collaert, et al. Effective dose range for dental cone beam computed tomography scanners. Eur J Radiol. 2012;81(2):267-271.

36. Liu L, Li J, Ji H, Zhang N, Wang Y, Zheng G, et al. Cone-beam computed tomography evaluation of the maxillofacial features of patients with unilateral temporomandibular joint ankylosis undergoing condylar reconstruction with an autogenous coronoid process graft. PLOS ONE. 2017;12(3):e0173142.

37. Gorucu-Coskuner H, Ciger S. Computed tomography assessment of temporomandibular joint position and dimensions in patients with class II division 1 and division 2 malocclusions. J Clin Exp Dent. 2017;9(3):e417423 .

38. Coskuner HG, Ciger S. Three-dimensional assessment of the temporomandibular joint and mandibular dimensions after early correction of the maxillary arch form in patients with Class II division 1 or division 2 malocclusion. Korean J Orthod. 2015;45(3):121-129.

39. Saione Cruz SA, Melo Saulo Leonardo Sousa, Melo Daniela Pita de, et al. Relationship between articular eminence inclination and alterations of the mandibular condyle: a CBCT study. Braz oral res. 2017;31:e25.

40. Sümbüllü MA, Cağlayan F, Akgül HM, et al. Radiological examination of the articular eminence morphology using cone beam CT. Dentomaxillofac Radiol. 2012;41(3):234-240.

41. Horner K, Islam M, Flygare L, et al. Basic principles for use of dental cone beam computed tomography: consensus guidelines of the European Academy of Dental and Maxillofacial Radiology. Dentomaxillofac Radiol. 2009;38(4):187-195.

42. Caruso S, Storti E, Nota A, et al. Temporomandibular Joint Anatomy Assessed by CBCT Images. BioMed Research International. 2017;2017:2916953.

43. Tabrizi R, Shahidi S, Bahramnejad E, et al. Evaluation of Condylar Position after Orthognathic Surgery for Treatment of Class II Vertical Maxillary Excess and Mandibular Deficiency by Using ConeBeam Computed Tomography. J Dent Shiraz Univ Med Sci. 2016;17(4):318325 .
44. Saati S, Kaveh F, Yarmohammadi S. Comparison of Cone Beam Computed Tomography and Multi Slice Computed Tomography Image Quality of Human Dried Mandible using 10 Anatomical Landmarks. $J$ Clin Diagn Res. 2017;11(2):ZC13-ZC16.

45. Al-Saleh M A, Punithakumar K, Lagravere M, et al. Three-dimensional morphological changes of the temporomandibular joint and functional effects after mandibulotomy. Journal of Otolaryngology - Head and Neck Surgery. 2017;46(8).

46. Ma RH, Yin S, Li G. The detection accuracy of cone beam CT for osseous defects of the temporomandibular joint: a systematic review and metaanalysis. Sci Rep. 2016;6:34714.

47. Al-Saleh MAQ, Jaremko JL, Alsufyani N, et al. Assessing the reliability of MRI-CBCT image registration to visualize temporomandibular joints. Dentomaxillofac Radiol. 2015;44(6):20140244.

48. Kyung-Soo Nah. Condylar bony changes in patients with temporomandibular disorders: a CBCT study. Imaging Sci Dent. 2012;42(4):249-253.

49. Naichuan Su, Yan Liu, Xianrui Yang, et al. Correlation between oral health-related quality of life and clinical dysfunction index in patients with temporomandibular joint osteoarthritis. J Oral Sci. 2016;58(4):483-490.

50. Jitka Levorová, Vladimír Machoň, Anasuya Guha, et al. Osteoarthrosis of Temporomandibular Joint Related to the Defects of Posterior Dentition: A Retrospective Study. Prague Med Rep. 2016;117(4):176-184.

51. Massilla Mani F, Satha Sivasubramanian S. A study of temporomandibular joint osteoarthritis using computed tomographic imaging. Biomed J. 2016;39(3):201-206.

52. Sung-Won Yang, Jin-Yong Cho, Hyeon-Min Kim. Septic arthritis of the temporomandibular joint: a case report. J Korean Assoc Oral Maxillofac Surg. 2016;42(4):227-230.

53. Gomes LR. Diagnostic index of 3D osteoarthritic changes in TMJ condylar morphology. Proc SPIE Int Soc Opt Eng. 2015;9414:81.

54. Anderson WM, Marsh CM, Kessel NC, et al. Studying the Prevalence and Etiology of Class II Subdivision Malocclusion Utilizing Cone-Beam Computed Tomography. J Orthod Endod. 2016;2:4.

55. Song Y, Zhang X, Yang Gao, et al. The condylar morphology in adult females of skeletal class II division 1 malocclusion with various vertical skeletal features: a study by cone beam computed tomography. Int J Clin Exp Med. 2016;9(5):8304-8311.

56. Singh A, Sangur R, Lakshmana BR, et al. Clinical Study to Determine the Pattern of Occlusal Contacts in Lateral Positions and Its Validity in Classifying Guidance Patterns. J Indian Prosthodont Soc. 2013;3(2):101107.

57. Gnanashanmugham K, Saravanan B, Sukumar MR, et al. Gnathological splint therapy in temporomandibular joint disorder. J Pharm Bioallied Sci. 2015;7(Suppl 1):S314-S318.

58. Larry $\mathrm{M}$ Wolford. Can orthodontic relapse be blamed on the temporomandibular joint? J Orthod Sci. 2014;3(4):95-105.

59. Ballanti F, Ranieri S, Baldini A, et al. Gnathological features in growing subjects. Annali di Stomatologia. 2013;IV(3-4):230-238.

60. Funato M, Ono Y, Baba K, et al. Evaluation of the non-functional tooth contact in patients with temporomandibular disorders by using newly developed electronic system. Journal of Oral Rehabilitation. 2014;41(3):170-176.

61. Kaidonis JA, Ranjitkar S, Lekkas D, et al. Functional dental occlusion: an anthropological perspective and implications for practice. Aust Dent J. 2014;59:(1 Suppl):162-173.

62. Felício CM, Melchior Mde O, Silva MA, et al. Masticatory performance in adults related to temporomandibular disorder and dental occlusion. Pró-Fono R. Atual Cient. 2007;19(2):151-197. 
63. Davies SJ, Gray RM, Sandler PJ, O'Brien KD. Orthodontics and occlusion. British Dental Journal. 2001;91(2):539-549.

64. Čimić S, Žaja M, Šimunković SK, et al. Influence of Occlusal Interference on the Mandibular Condylar Position. Acta Stomatol Croat. 2016;50(2):116-121.
65. Skirbutis G, Surna A, Barauskas R, et al. A revised approach of human mastication function rehabilitation through monotypical mastication analysis. Stomatologija. 2015;17(1):13-20. 